\title{
The why and wherefore of hepatic encephalopathy
}

This article was published in the following Dove Press journal:

International Journal of General Medicine

16 December 2015

Number of times this article has been viewed

Vijay PB Grover

Joshua M Tognarelli

Nicolas Massie

Mary ME Crossey

Nicola A Cook

Simon D Taylor-Robinson

Liver Unit, Division of Diabetes, Endocrinology and Metabolism, Department of Medicine, Imperial College London, London, UK
Correspondence: Joshua M Tognarelli Liver Unit, Division of Diabetes, Endocrinology and Metabolism,

Department of Medicine, I0th Floor Queen Elizabeth the Queen Mother Wing, St Mary's Hospital Campus, Imperial College London, Praed Street, London W2 INY, UK

Tel +44207886 6454

$\mathrm{Fax}+442074022796$

Email jt21 I0@imperial.ac.uk
Abstract: Hepatic encephalopathy is a common neuropsychiatric abnormality, which complicates the course of patients with liver disease. It was probably first described by Hippocrates over 2000 years ago, who said that "those whose madness arises from phlegm are quiet and neither shout nor make a disturbance, while those whose madness arises from bile shout, play tricks and will not keep still, but are always up to some mischief". He was presumably describing the differences between patients with pneumonia and acute liver failure. Despite the fact that the syndrome was probably first recognized thousands of years ago, the exact pathogenesis still remains unclear. Furthermore, a precise definition of the syndrome is lacking, as are definitive methods of diagnosing this condition. It is important as both patients with cirrhosis and the general population with whom they interact may be affected as a consequence. At a minimum, the individual may be affected by impaired quality of life, impaired ability to work, and slowed reaction times, which are relevant to the population at large if affected individuals operate heavy machinery or drive a car. Pathogenic mechanisms, diagnostic tools, and treatment options are discussed.

Keywords: hepatic encephalopathy, cirrhosis, ammonia, pathology, treatment, rifaximin, lactulose

\section{Background}

Hepatic encephalopathy (HE) is a complex, reversible neuropsychiatric syndrome, complicating the course of liver disease. In recent guidelines published jointly by the European and American Associations for the Study of the Liver, HE was defined as "brain dysfunction caused by liver insufficiency or portal systemic shunting". ${ }^{1,2}$ Despite the fact that the syndrome was probably recognized thousands of years ago, the exact pathogenesis remains unclear. ${ }^{3}$

The pathogenesis is thought to be attributable to both neurochemical and neurophysiological disorders of the brain. ${ }^{4}$ The essentially reversible nature suggests a metabolic cause. The broad spectrum of cerebral disturbance is likely to be a reflection of the range of metabolic disturbances responsible for the syndrome, rather than one causal abnormality.

One of the central factors contributing to $\mathrm{HE}$ in cirrhosis is unfiltered blood from the portal system reaching the brain. Neuropathologically, the most frequently described change is to astrocytes, which undergo cell swelling. ${ }^{5}$ The morphological change seen is known as Alzheimer type II astrocytosis. However, neurons remain structurally normal. Theories on pathogenesis of HE are outlined in Table 1. 
Table I Theories for pathogenesis of HE

\begin{tabular}{|c|c|}
\hline Location of alteration & Suggested mechanism \\
\hline \multirow[t]{3}{*}{ Gut-derived neurotoxins } & Ammonia \\
\hline & Short- and medium-chain fatty acids \\
\hline & Amino acid disturbances \\
\hline \multirow[t]{3}{*}{ Cerebral neurotransmission } & GLU \\
\hline & GABA \\
\hline & Peripheral benzodiazepine binding sites \\
\hline \multirow[t]{2}{*}{ Cerebral energy metabolism } & Cerebral blood flow changes \\
\hline & Alteration in cerebral glucose metabolism \\
\hline Blood-brain barrier & Functional changes \\
\hline Low-grade cerebral edema & Astrocyte swelling \\
\hline
\end{tabular}

Abbreviations: $\mathrm{HE}$, hepatic encephalopathy; GLU, glutamate; GABA, $\gamma$-aminobutyric acid.

\section{Gut-derived neurotoxins}

Ammonia has been the most studied gut-derived neurotoxin, produced from the breakdown of proteins and amino acids. Ammonia is produced by the gastrointestinal tract in two ways: by direct ammonia liberation from breakdown products of dietary protein and metabolism of circulating glutamine (GLN) and by the gut microbiome acting upon urea and ingested food. Concentrations of ammonia are kept relatively constant in the blood by efficient detoxification processes, involving hepatic production of urea and synthesis of GLN from glutamate (GLU) by the action of glutamine synthetase (GS), which is located in the liver, muscle, and brain. ${ }^{6}$ Hyperammonemia is commonly seen in chronic liver disease, as are high levels of circulating endotoxins, as the liver fails to detoxify the portal circulation draining the intestines, which are heavily colonized by metabolically active bacteria, or else because the portal blood supply bypasses the liver through the development of a collateral circulation in the presence of portal hypertension.

The first suggestion that ammonia may be involved in HE was in 1893 by investigators from Pavlov's group. ${ }^{7}$ A rise in arterial blood ammonia in dogs was associated with behavioral disturbances after high protein meals. ${ }^{8}$ Fifty years later, Gabuzda et al inadvertently produced $\mathrm{HE}$ in chronic liver disease patients, treated with ion-exchange resins to diminish ascites. ${ }^{9}$ The resins absorbed sodium, but released ammonium ions, bringing about HE.

More recent animal studies using portacaval shunts showed blood and brain ammonia concentrations to be increased two- and three folds, respectively. ${ }^{10}$ Primary cultured astrocytes exposed to ammonia have been shown to develop features of Alzheimer type II astrocytosis seen neuropathologically in human beings. ${ }^{11}$

Short- and medium-chain fatty acids are increased in the blood of patients with $\mathrm{HE} .^{12}$ These fatty acids produce coma in animal models and possibly interfere with ureagenesis. ${ }^{13}$
Nevertheless, these agents have been given to patients without any adverse consequences. ${ }^{14}$

Amino acid disturbances have been observed in HE patients, typically increased aromatic amines and decreased or normal levels of branched-chain amino acids (BCAAs). ${ }^{15,16}$ Low plasma concentrations of BCAAs are a result of increased use by muscle, heart, kidney, and adipose tissue as an energy source, ${ }^{17}$ since alternatives such as glucose and ketone bodies are reduced in liver disease. Hyperammonemia results in increased brain uptake of aromatic acids, including tryptophan, the precursor of the neurotransmitter, serotonin. Tryptophan's increased availability leads to altered serotonin synthesis, increasing neuroinhibition. ${ }^{18}$

\section{Cerebral neurotransmission}

GLU is the predominant excitatory neurotransmitter in the brain. In HE, astrocytes play an important role in detoxification of ammonia through conversion of GLU to GLN. Astrocytic GLU reuptake mechanisms may become impaired; thereby increasing extracellular concentrations of GLU. ${ }^{19}$ Furthermore, GLU binding sites appear to be downregulated on postsynaptic neurons. This, therefore, contributes to decreased neuroexcitatory activity ${ }^{20}$ and increased cerebral GLN. ${ }^{21}$

$\gamma$-Aminobutyric acid (GABA) is the predominant inhibitory neurotransmitter in the brain, ${ }^{22}$ binding to specific GABA receptors, which are part of larger receptor complexes, activated by benzodiazepines and barbiturates. For the most part, GABA is synthesized by gut bacteria and would normally enter the portal vein to be metabolized by the liver. In cirrhosis, blood bypasses the liver by collaterals and enters the systemic circulation. Increased plasma levels of GABA have been observed in patients with HE. ${ }^{23}$ However, autopsied brain tissue from HE patients did not support GABA involvement. ${ }^{24}$

Translocator proteins (TSPOs), formerly known as peripheral benzodiazepine binding sites, may play an important role in neuroinhibition. TSPOs are $18 \mathrm{kDa}$ proteins, located on the outer mitochondrial membrane of astrocytes, and facilitate cholesterol ingress into mitochondria, among other roles. ${ }^{25}$ TSPOs are widely distributed throughout the body and are also present in microglia. ${ }^{26}$ TSPOs are upregulated by ammonia ${ }^{27}$ and have been shown to be present in increased density in autopsied brains of HE patients. ${ }^{28}$ TSPO upregulation promotes synthesis of neurosteroids, such as tetrahydroprogesterone and tetrahydrodeoxycorticosterone, which are potent agonists of $\mathrm{GABA}_{\mathrm{A}}$ receptors (Figure 1). In vivo positron emission tomography (PET) studies support the hypothesis that TSPOs could play an active role in impaired brain functioning in $\mathrm{HE}^{29}$ 


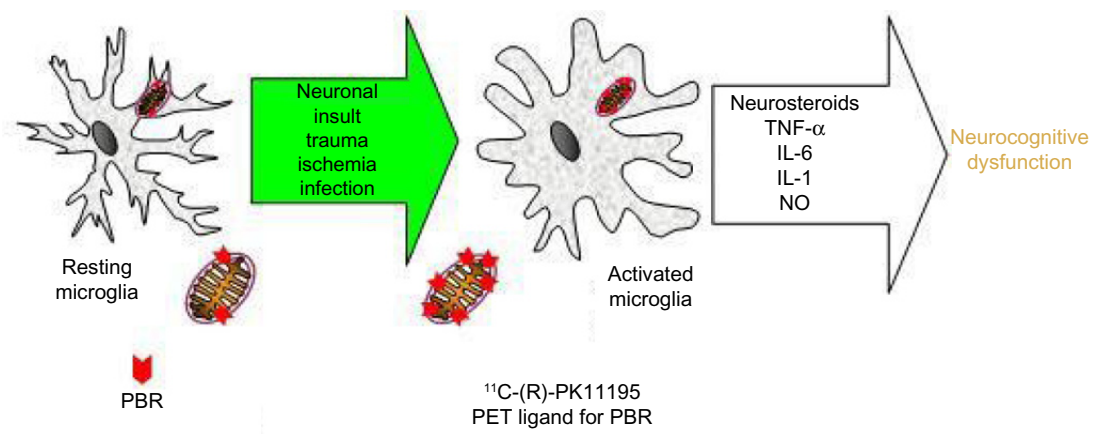

Figure I Schematic representation of microglial activation.

Notes: Resting microglia are spindly and spiky in appearance on electron microscopy, demonstrating low levels of constitutive PBR binding site activity (TSPO) on their mitochondria. When "activated", microglia demonstrate an increased number of PBR binding sites. "C-(R)-PKIII 95 is a PET ligand that binds to PBRs.

Abbreviations: PBR, peripheral benzodiazepine receptor; TSPO, translocator protein; PET, positron emission tomography; TNF- $\alpha$, tumor necrosis factor-alpha; IL, interleukin; NO, nitric oxide.

\section{Inflammation}

It is frequently observed that cirrhosis patients with active infections may exhibit HE, leading several investigators to consider a direct link between HE and inflammation. One group medically induced hyperammonemia, using amino acid solution, in a group of stable cirrhosis patients. ${ }^{30}$ Patients with abnormal psychometric performance had more markedly elevated inflammatory markers (white cell count, neutrophil count, C-reactive protein, nitrate/nitrite, and interleukin [IL]-6). Psychometric performance deteriorated in $44.8 \%$ of patients with the amino acid solution. In "deteriorators", there was an increase in inflammatory markers, compared to "nondeteriorators". Further support for this theory is provided by another group, which studied effects of alteration of gut flora with administration of fiber and probiotics upon HE patients. ${ }^{31}$ They showed that improvement in HE was associated with reductions in venous endotoxin and ammonia levels. This is an area of increasing interest that requires further investigation.

\section{Low-grade cerebral edema}

It has been proposed by Häussinger et al that a major contributory event to the development of HE in patients with chronic liver disease is an increase in astrocyte hydration (low-grade cerebral edema without a clinically overt increase in intracranial pressure).$^{32}$ This may occur as a consequence of astrocytic ammonia uptake and subsequent detoxification by GS, forming GLN from GLU. ${ }^{10}$ This deamidation process is thought to result in accumulation of GLN within astrocytes, acting as a cerebral osmolyte. The cells respond by expelling osmolytes, myo-inositol (mI), choline (cho), and GLN, but the membrane transport systems are not able to keep up with greatly increased production of GLN. Equilibrium cannot be re-established and there is resultant cell swelling (Figure 2).
Cerebral magnetic resonance spectroscopy (MRS) studies in vivo demonstrated reduced levels of $\mathrm{mI}$ and increased GLN and GLU (Glx). ${ }^{33}$ Additionally, benzodiazepines, hyponatremia, and inflammatory cytokines can induce astrocyte swelling in vitro, so these factors may act synergistically with ammonia to produce low-grade cerebral edema. ${ }^{32}$

\section{Clinical features}

Recognized clinical features of HE are listed in Table 2. The first widely accepted clinical grading system to attempt to categorize HE was described by Parsons-Smith et al. ${ }^{34}$ This classification system was later modified and is now known as either the "modified Parsons-Smith" or West Haven criteria $^{35}$ (Table 3).

\section{Minimal hepatic encephalopathy}

The first published work to propose that subtle mental changes can occur before the onset of clinically detectable neurological changes was by Zeegen et al in 1970. ${ }^{36}$ They performed paper-based psychometric tests, consisting of the construction of five-pointed stars and modified "Reitan" trail making tests on a population of patients after portal decompression surgery. They found that 13 out of 34 patients (38\%), without clinical signs of HE on standard clinical examination, had impaired performance on Reitan trail making tests.

\section{Nomenclature}

Subsequently, the presence of these subtle abnormalities has been observed by numerous investigators and gaged by a variety of psychometric tests. ${ }^{37,38}$ The presence of this abnormality was first termed subclinical or latent HE owing to the lack of clinical signs on examination. Subsequently, nomenclature was replaced by the term "minimal" HE 


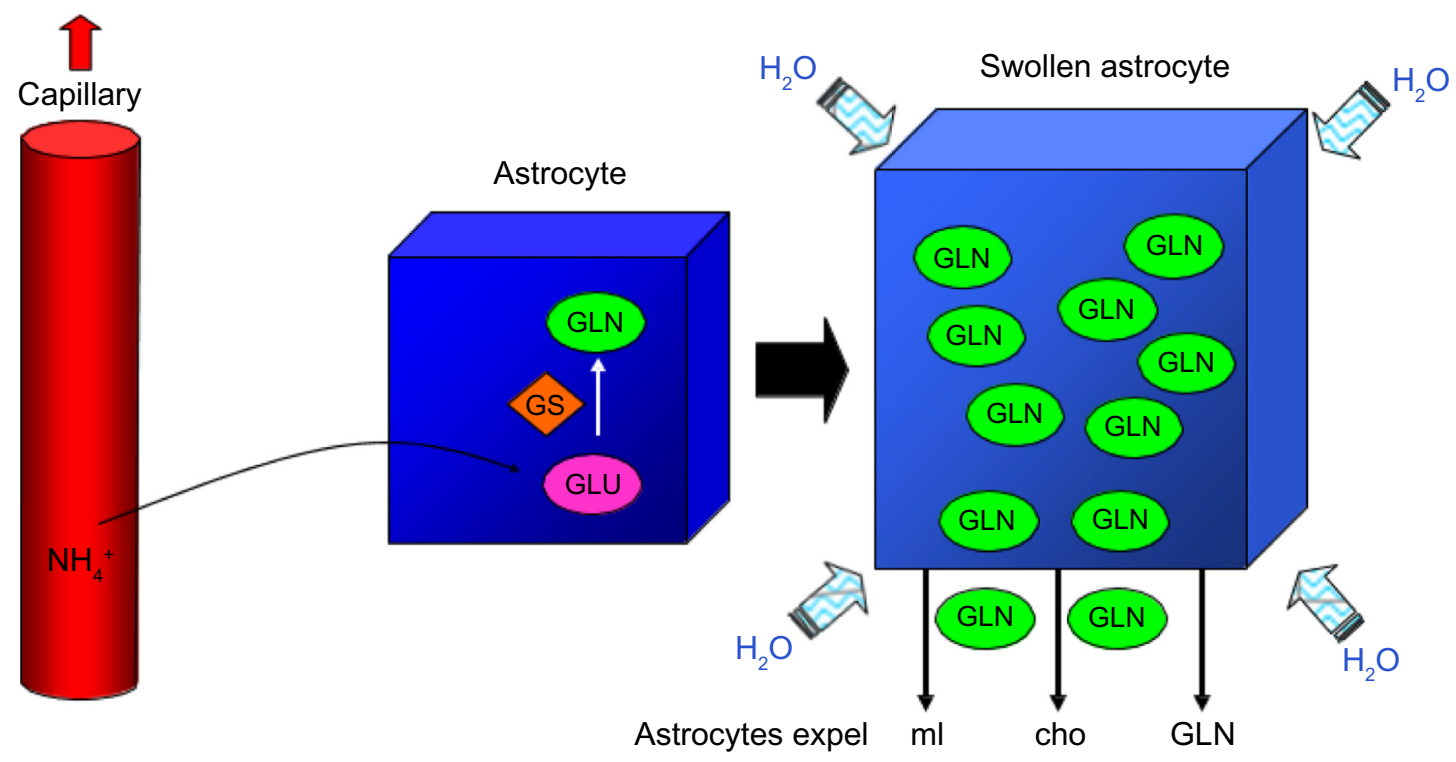

Figure 2 Hypothesis of astrocyte swelling contributing to low-grade cerebral edema.

Abbreviations: GLN, glutamine; GS, glutamine synthetase; GLU, glutamate; ml, myo-inositol; cho, choline.

$(\mathrm{mHE})^{39}$ (Table 4). To confuse matters further, the 2014 AASLD/EASL guidelines also suggest an alternative classification considering mHE (West Haven grade 0) and West Haven grade 1 overt HE as "covert" HE, while West Haven grade 2 and above are considered as "overt" HE. ${ }^{1}$ However, this classification is rarely used outside the United States.

\section{Clinical significance}

mHE has been shown to have effects upon quality of life, earning potential, driving performance, and possibly survival.

\section{Quality of life}

Groeneweg et al used the "Sickness Impact Profile" (SIP) to determine the effects of $\mathrm{mHE}$ on patients' "activities

Table 2 Recognized features of $\mathrm{HE}$

\begin{tabular}{ll}
\hline Affected domain & Symptom/sign \\
\hline Disturbed consciousness & Reversal of sleep pattern \\
& Drowsiness \\
& Coma (later stages) \\
Personality changes & Irritability \\
& Irrational behavior \\
Intellectual impairment & Reduced attention span \\
& Impaired mental agility \\
& Impaired working memory \\
Speech & Slow \\
& Slurred \\
Motor & Asterixis/flapping tremor \\
& Ataxic gait \\
\hline
\end{tabular}

Abbreviation: HE, hepatic encephalopathy. of daily living". ${ }^{40}$ The SIP is a questionnaire assessing influence of the disease and treatment on daily functioning. ${ }^{41}$ The questionnaire consists of 136 items, grouped into 12 scales encompassing different aspects of daily living, eg, sleep, eating, social interaction, and emotional behavior. They found that $\mathrm{mHE}$ patients performed significantly worse in every dimension of the SIP. ${ }^{40}$

\section{Earning potential}

Schomerus and Hamster selected 110 ambulatory outpatients with cirrhosis, who were part of a larger study undergoing psychometric testing. ${ }^{42}$ Of white-collar workers, $40 \%$ had abnormal psychometry, but only $20 \%$ were unable to continue

Table 3 Modified Parsons-Smith or West Haven criteria for HE

\begin{tabular}{ll}
\hline Grade 0 & No abnormality detected (mHE) \\
Grade I & Trivial lack of awareness \\
& Euphoria or anxiety \\
& Shortened attention span \\
& Impairment of addition or subtraction \\
& Lethargy \\
Grade 2 & Disorientation for time \\
& Obvious personality change \\
& Inappropriate behavior \\
& Somnolence to semistupor \\
Grade 3 & Responsive to stimuli \\
& Confused \\
& Gross disorientation, bizarre behavior \\
& Coma, unable to test mental state
\end{tabular}

Note: This article was published in Gastroenterology, vol 72, Conn HO et al, 1977, Comparison of lactulose and neomycin in the treatment of chronic portal-systemic encephalopathy. A double blind controlled trial, pp573 to $583 .{ }^{35}$ Copyright Elsevier (c) 2013.

Abbreviations: $\mathrm{HE}$, hepatic encephalopathy; $\mathrm{mHE}$, minimal $\mathrm{HE}$. 
Table 4 Proposed nomenclature of HE

\begin{tabular}{|c|c|c|c|}
\hline HE type & Nomenclature & Subcategory & Subdivisions \\
\hline A & Associated with acute liver failure & & \\
\hline B & Associated with portal-systemic bypass and no intrinsic hepatocellular disease & & \\
\hline \multirow[t]{7}{*}{ C } & Associated with cirrhosis and portal hypertension or portal-systemic shunts & Episodic HE & Precipitated \\
\hline & & & Spontaneous \\
\hline & & & Recurrent \\
\hline & & Persistent HE & Mild \\
\hline & & & Severe \\
\hline & & & Treatment dependent \\
\hline & & $\mathrm{mHE}$ & \\
\hline
\end{tabular}

Note: Copyright John Wiley \& Sons (c) 2003. Reproduced from Ferenci P. Hepatic encephalopathy - definition, nomenclature, diagnosis, and quantification: final report of the working party at the I I th World Congresses of Gastroenterology, Vienna, I998. Hepatology. 2002;35(3):7I6-72I..$^{39}$

Abbreviations: $\mathrm{HE}$, hepatic encephalopathy; $\mathrm{mHE}$, minimal $\mathrm{HE}$.

working, whereas $55 \%$ of blue-collar workers had abnormal psychometry and $60 \%$ were unable to earn their living. ${ }^{42}$

\section{Driving ability}

Wein et al measured "on-the-road" driving performance. Of 274 consecutive patients with cirrhosis, 48 fulfilled medical and driving inclusion criteria, 14 of them with $\mathrm{mHE}$ and 34 without mHE. The likelihood of an accident in mHE patients was nearly ten times higher than that of patients without mHE. ${ }^{43}$ Bajaj et al studied self-reported driving behavior in postal questionnaires. Patients with cirrhosis had a higher percentage of driving violations over the previous year (13\%), previous 5 years $(25 \%)$, and a higher number of accidents at 1 year $(9 \%)$ and 5 years $(17 \%))^{44}$

\section{Diagnosis}

Despite agreement between investigators that mHE warrants further investigation and probably screening and treatment, there is little consensus as to the optimal instruments with which to diagnose and monitor the condition. ${ }^{39}$ This has a significant role in the discrepancy between studies, as to the prevalence of mHE, with quoted prevalence varying from $27 \%$ to $75 \%$ according to the battery of tests used, interpretation of these tests, and populations studied. ${ }^{45,46}$ Some studies have used just one psychometric test,${ }^{47}$ whereas other studies have used up to 26 tests. ${ }^{38}$ In the latter case, tests needed to be spread over 2 days to avoid fatigue.

In response to the variability of diagnostic tests being used to define mHE, a consensus was reached by a working party, ${ }^{39}$ which proposed use of a psychometric hepatic encephalopathy score, based on the results of five neuropsychometric tests. ${ }^{48}$ They also suggested that when possible, quantitative neurophysiological tools, such as electroencephalography (EEG) with mean dominant frequency and P300 auditory evoked potentials, should be used. ${ }^{1,2}$

\section{Assessment of HE}

HE affects cognitive, affective/emotional, behavioral, and bioregulatory domains..$^{39}$ Each broad domain may be subdivided into various components. For example, cognition may include evaluation of psychomotor speed, visuopraxis, attention, concentration, and level of consciousness. ${ }^{39}$ Overt or clinically apparent HE should be excluded by careful and detailed neuropsychiatric examination and anamnestic enquiry. Particular attention should be paid to cognitive and motor function, ability to perform activities of daily living, and sleep-wake cycle abnormalities. To diagnose mHE, at least two neuropsychological tests from the following psychometric hepatic encephalopathy score system should be used:

1. Number connection test-A (NCT-A);

2. NCT-B; ${ }^{49}$

3. Block design test;

4. Digit symbol substitution test. ${ }^{50}$

The recommendation was to use a standardized battery including NCT-A and NCT-B, the line tracing, serial dotting, and digit symbol substitution tests. ${ }^{1,2}$ This battery is easy to apply, but reference test scores are normalized for German populations, so it may be necessary to adjust these for other patient populations. Reference ranges are now available for Spanish, ${ }^{51}$ Italian, and British populations. ${ }^{52}$

\section{Paper-based tests}

The theoretical advantages of paper-based tests are that they are easy to administer, require no sophisticated technical equipment, and can be performed at the bedside. However, in practice, they may be subject to a greater degree of subjectivity. Ideally, they should be performed in a quiet, well-lit room, under standardized conditions, which apply to both examiner and examinee. In practice, these conditions do not exist in hospital wards. The paper-and-pencil tasks may be affected by subjective "one-off influences", such as lack of sleep, recent arguments, or hunger. Theoretically, 
the tests should also be easily interpretable, but reference scores should take account of education, cultural background, and language difficulties.

\section{Quality of life scores}

Other paper-based assessments of mHE include nonpsychometric tests such as validated quality of life scores, such as the short form 36 health survey, SIP, and fatigue impact questionnaires. $^{53-55}$

\section{Computer-based psychometric tests}

The use of computerized psychometric testing is widespread in both clinical research settings and for monitoring effects of pharmacological compounds upon cognitive function. ${ }^{56}$ The choice of system varies from center to center, depending upon investigators' personal experience, developmental input, and financial restraints. The advantages of computerized systems above conventional paper-and-pencil tests include reproducibility of stimulus presentation, accurate recording of responses/reaction times, and easier to facilitate data analysis. Furthermore, some tests are entirely visual and therefore do not depend on literacy and numeracy, which can hamper the interpretation of paper-based tests. For example, the use of the Stroop test on smartphones and computer tablets has proven to be an easily accessible, useful tool in many countries of the developed world. ${ }^{57}$

\section{Electroencephalography}

The EEG detects and records patterns of electrical activity within the brain through electrodes placed in multiple areas on the scalp. EEG abnormalities in hepatic coma were described in $1950 .{ }^{58}$ One of the more constant features in the studies of $\mathrm{HE}$ is the presence of a generalized slowing of background EEG activity, which appears to be a constant, progressive, and a quantifiable finding. It is important to note that EEG slowing is nonspecific as it is observed in other metabolic and drug-induced encephalopathies. ${ }^{4}$

\section{Evoked potentials}

Evoked potentials are electrical potentials, elicited in conjunction with sensory, motor, and cognitive events. They are a measure of the conduction and function of afferent pathways between stimulated peripheral nerves and the cortex. Abnormalities of visual evoked potentials, brainstem auditory evoked potentials, and somatosensory evoked potentials have been demonstrated in HE, but the sensitivity of the techniques appear to vary significantly between studies. ${ }^{4} \mathrm{P} 300$ is a cognitive evoked potential elicited when a subject receives an infrequent stimulus embedded within a group of irrelevant stimuli (oddball paradigm). The subject is asked to identify the oddball stimulus and a potential is elicited independent of the sensory modality being stimulated. ${ }^{4}$ The potential occurs $\sim 300 \mathrm{~ms}$ after the stimulus, hence its name. The P300 appears to be useful as an investigative research tool in lower grades of $\mathrm{HE}^{4}$

\section{Critical flicker frequency}

The critical flicker frequency (CFF) of an individual is the highest frequency in Hertz that an individual perceives the "flicker" of a flickering light. All frequencies above that threshold will be observed to be continuous. CFF is thought to reflect both the efficiency of the visual pathways and the cerebral cortex. A single study has used CFF in a wellcharacterized cohort of patients with cirrhosis and healthy controls. ${ }^{59}$ CFF thresholds were found to be significantly lower in patients with $\mathrm{mHE}$ and overt HE than in healthy controls or unimpaired patients. Furthermore, they were able to define a threshold value of $39 \mathrm{~Hz}$, which divided impaired from unimpaired patients.

\section{Brain imaging}

Currently, there is no established imaging modality or technique for the assessment of HE in clinical practice. The most widely utilized modality is magnetic resonance imaging (MRI), with the most consistently reported sequence being MRS. MRS is a noninvasive technique used to determine the biochemical profile of a region of interest in vivo, thus giving information regarding the metabolic processes within that region. Several investigators have found consistent abnormalities in the MRS data from patients with HE. ${ }^{60}$

Other imaging modalities, such as PET, have been used in one case series, ${ }^{29}$ but have not been replicated by other groups. Similarly, techniques such as magnetoencephalography have been described in the research setting, ${ }^{61}$ but worldwide, very few centers have this equipment available.

\section{Treatment of hepatic encephalopathy}

No truly specific treatment for HE exists, as the exact pathogenesis is unknown. The pharmacological treatments that are currently used in clinical practice are directed toward reducing the production and absorption of gut-derived ammonia.

\section{Antibiotics}

Antibiotics have been used to treat HE since the $1950 \mathrm{~s}^{62}$ The nonabsorbable antibiotic, neomycin, reduces bacterial 
ammonia production in the colon, by altering the normal gut flora. Neomycin is considered to be nonabsorbable, but a small percentage is absorbed, causing ototoxic and nephrotoxic side effects. ${ }^{63}$ Neomycin is now rarely used as rifaximin, which lacks these side effects, has become available more recently.

Rifaximin is a nonabsorbable derivative of rifamycin with a broad spectrum of activity against aerobic and anaerobic gram-positive and gram-negative organisms. It has a bioavailability of $<0.4 \%$, and $\sim 97 \%$ of the drug is excreted in feces unchanged. ${ }^{64}$ These pharmacokinetic properties make it an attractive option for the treatment of HE. Clinical trials have compared rifaximin to lactulose or neomycin for treatment of HE. Rifaximin demonstrates a general trend toward better efficacy, compared with lactulose or neomycin, but long-term safety data are lacking. ${ }^{65}$ More recent papers have found rifaximin to be highly effective in the management of HE for the treatment, maintenance of remission, and reduction in hospitalizations of chronic liver disease patients. ${ }^{66,67}$ However, the recent EASL/AASLD guidelines only recommend its use as an add-on therapy to lactulose, not as a first-line or sole treatment agent. ${ }^{1,2}$

\section{Lactulose}

Lactulose is a synthetic disaccharide taken orally, primarily used to treat constipation. It has been used to treat $\mathrm{HE}$ since the 1960s. ${ }^{68}$ When it reaches the colon, lactulose is metabolized by bacteria, predominantly to lactic acid. The fecal pH drops, favoring growth of lactose-fermenting organisms and suppressing organisms such as Bacteroides spp., which are ammonia formers. Additionally, it is thought that fecal acidity reduces ionization and subsequent absorption of ammonia. It has been demonstrated that lactulose at least doubles the colonic output of bacterial mass and soluble nitrogen, which can then no longer be absorbed. ${ }^{69}$ Side effects of treatment include flatulence, diarrhea, and abdominal pain.

\section{L-Ornithine L-aspartate}

L-Ornithine L-aspartate (LOLA) is a treatment directed at removal of circulating ammonia. Ornithine promotes hepatic removal of ammonia by stimulating residual hepatic urea cycle activity through action of ornithine carbamoyltransferase and carbamoylphosphate synthetase. Additionally, ornithine and aspartate are both substrates for the urea cycle. In perivenous hepatocytes, ornithine and aspartate combine with $\alpha$-ketoglutarate to produce GLU. GLU is used by skeletal muscle and brain to use ammonia, via the action of GS to produce GLN, reducing the amount of circulating ammonia. ${ }^{70}$ One study has demonstrated that intravenous LOLA ameliorates deleterious psychometric effects of GLN in Child's grade B and C patients. ${ }^{71}$ A study of overt HE patients randomized to receive either oral LOLA or lactulose for 2 weeks found that while both agents reduced circulating ammonia levels, use of LOLA was also associated with significant improvements in mental status, psychometric parameters, and EEG activity. ${ }^{72}$ A meta-analysis of three randomized, controlled studies of the LOLA use in 212 patients with HE concluded that LOLA is of benefit in overt $\mathrm{HE}$, but the currently available data do not support its use in $\mathrm{mHE}{ }^{73}$

\section{Nutritional therapies Vegetable-based protein}

Contrary to previous reports, patients with HE should not be protein restricted. They are often in a catabolic state and require $1-1.5 \mathrm{~g} / \mathrm{kg}$ of protein per day. Vegetable protein tends to be better tolerated than meat protein. Additionally, due to their high fiber content, vegetable proteins increase colonic motility, as well as improve fecal nitrogen output. ${ }^{74,75}$ However, the clinical utility of a vegetable protein-based diet is limited by poor patient compliance and is not used routinely in many centers.

\section{Prebiotics, probiotics, and symbiotics}

Prebiotics are nondigestible food ingredients, stimulating growth of select colonic bacteria to improve host health, whereas probiotics are live microbial food supplement improving host gut microbial balance. Symbiotics are a combination of the two. A meta-analysis demonstrated that supplementing the diet with pre-, pro-, and symbiotics can lead to significant improvements in patients with MHE, suggesting that their inclusion may have clinical benefits. Furthermore, the supplements were well tolerated. ${ }^{76}$

\section{Branched-chain amino acids}

Administration of preparations high in BCAAs and low in aromatic amino acids (AAAs) is based on reducing transmission of AAAs across the blood-brain barrier, thus reducing production of false neurotransmitters, which can contribute to encephalopathy. ${ }^{75}$ Although BCAAs have been shown to improve both recovery and duration of hospital admissions, ${ }^{77}$ a Cochrane systematic review found that BCAAs do not have significant beneficial effects on patients with HE. Studies involving BCAAs tend to be limited by short follow-up periods and poor methodological quality. ${ }^{78}$ Higher quality studies are required in this area. 


\section{Orthotopic liver transplantation}

Orthotopic liver transplantation (OLT) is the definitive treatment for HE arising in both acute liver failure (ALF) and chronic liver disease (CLD). However, its urgency depends on the cause and nature of liver failure. In patients with ALF who are progressing to cerebral edema and HE, an OLT is crucial to survival and urgent discussion with a liver transplant center is required to prevent deterioration to cerebral coning and brain death. In patients with CLD, HE tends to be chronic and fluctuating. It is considered to be one of the cardinal manifestations of decompensating liver disease. OLT in this group of patients tends to be determined according to need and prospective survival via established CLD criteria. ${ }^{79}$

\section{Conclusion}

HE is a neuropsychiatric syndrome, with symptoms existing on a continuum. Early recognition and management is imperative in optimizing outcome, particularly in the context of the timing of OLT. With the right treatment, most patients with overt HE can lead relatively normal lives with reasonable neuropsychological function. Stability of underlying liver function and prompt treatment of precipitating factors, such as variceal bleeding and infections, is crucial to this. However, it should be remembered that in the acute setting, HE is the hallmark of acute liver failure and that urgent OLT may be required.

\section{Acknowledgment}

All the authors acknowledge the support of the National Institute for Health Research Biomedical Research Centre at Imperial College London for infrastructure support.

\section{Disclosure}

The authors report no conflicts of interest in this work.

\section{References}

1. American Association for the Study of Liver, European Association for the Study of the Liver. Hepatic encephalopathy in chronic liver disease: 2014 practice guideline by the European Association for the study of the liver and the American Association for the study of liver diseases. J Hepatol. 2014;61(3):642-659.

2. Vilstrup H, Amodio P, Bajaj J, et al. Hepatic encephalopathy in chronic liver disease: 2014 practice guideline by the American Association for the study of liver diseases and the European Association for the study of the liver. Hepatology. 2014;60(2):715-735.

3. Häussinger D, Schliess F. Pathogenetic mechanisms of hepatic encephalopathy. Gut. 2008;57(8):1156-1165.

4. Montagnese S, Amodio P, Morgan MY. Methods for diagnosing hepatic encephalopathy in patients with cirrhosis: a multidimensional approach. Metab Brain Dis. 2004;19(3):281-312.

5. Butterworth RF. Pathophysiology of hepatic encephalopathy: a new look at ammonia. Metab Brain Dis. 2002;17(4):221-227.
6. Shawcross D, Jalan R. The pathophysiologic basis of hepatic encephalopathy: central role for ammonia and inflammation. Cell Mol Life Sci. 2005;62(19):2295-2304.

7. Hahn M, Massen O, Nencki M, Pavlov I. Die Eck'sche fistel zwischen der unteren hohlvene und der pfortader und ihre folgen fur den organismus. Arch Exp Pathol Pharm. 1893;32:161-210.

8. Nencki M, Pawlow JP, Zalenski J. Ueber den ammoniakgehalt des blutes und der organe und die harnstoff- bildung bei den saugethieren [On the subject of ammonia content of blood and organs and urea formation in mammals]. Archiv Fuer Experimentelle Pathologie und Pharmakologie. 1896;37:26-51. German.

9. Gabuzda G, Phillips G, Davidson C. Reversible toxic manifestations in patients with cirrhosis of the liver given cation-exchange resins. N Engl J Med. 1952;246(4):124-130.

10. Butterworth R, Giguère J, Michaud J, Lavoie J, Layrargues G. Ammonia: key factor in the pathogenesis of hepatic encephalopathy. Neurochem Pathol. 1987;6(1-2):1-12.

11. Gregorios J, Mozes L, Norenberg M. Morphologic effects of ammonia on primary astrocyte cultures. II. Electron microscopic studies. J Neuropathol Exp Neurol. 1985;44(4):404-414.

12. Chen S, Mahadevan V, Zieve L. Volatile fatty acids in the breath of patients with cirrhosis of the liver. J Lab Clin Med. 1970;75(4): 622-627.

13. Zieve L, Doizaki WM, Zieve J. Synergism between mercaptans and ammonia or fatty acids in the production of coma: a possible role for mercaptans in the pathogenesis of hepatic coma. J Lab Clin Med. 1974;83(1):16-28.

14. Morgan M, Bolton C, Morris J, Read A. Medium chain triglycerides and hepatic encephalopathy. Gut. 1974;15(3):180-184.

15. Fischer J, Yoshimura N, Aguirre A, et al. Plasma amino acids in patients with hepatic encephalopathy. Am J Surg. 1974;127(1):40-47.

16. Iber FL, Rosen H, Levenson SM, Chalmers TC. The plasma amino acids in patients with liver failure. J Lab Clin Med. 1957;50(3):417-425.

17. Soeters P, Weir G, Ebeid A, Fischer J. Insulin, glucagon, portal systemic shunting, and hepatic failure in the dog. J Surg Res. 1977;23(3):183-188

18. Curzon G, Kantamaneni B, Fernando J, Woods M, Cavanagh J. Effects of chronic porto-caval anastomosis on brain tryptophan, tyrosine and 5-hydroxytryptamine. J Neurochem. 1975;24(5):1065-1070.

19. Moroni F, Lombardi G, Moneti G, Cortesini C. The release and neosynthesis of glutamic acid are increased in experimental models of hepatic encephalopathy. J Neurochem. 1983;40(3):850-854.

20. Raabe W. Synaptic transmission in ammonia intoxication. Neurochem Pathol. 1987;6(1-2):145-166.

21. Hourani B. Cerebrospinal fluid glutamine as a measure of hepatic encephalopathy. Arch Intern Med. 1971;127(6):1033.

22. Basile A, Jones E. Ammonia and GABA-ergic neurotransmission: interrelated factors in the pathogenesis of hepatic encephalopathy. Hepatology. 1997;25(6):1303-1305.

23. Levy LJ, Leek J, Losowsky MS. Evidence for gamma-aminobutyric acid as the inhibitor of gamma-aminobutyric acid binding in the plasma of humans with liver disease and hepatic encephalopathy. Clin Sci (Lond). 1987;73(5):531-534.

24. Sherlock S, Dooley J. Diseases of the Liver and Biliary System. 11 ed. Oxford: Blackwell Science; 2002.

25. Anholt RR, Pedersen PL, De Souza EB, Snyder SH. The peripheraltype benzodiazepine receptor. Localization to the mitochondrial outer membrane. J Biol Chem. 1986;261(2):576-583.

26. Banati R. Visualising microglial activation in vivo. Glia. 2002;40(2): 206-217.

27. Itzhak Y, Norenberg M. Ammonia-induced upregulation of peripheraltype benzodiazepine receptors in cultured astrocytes labeled with [3H] PK 11195. Neurosci Lett. 1994;177(1-2):35-38.

28. Lavoie J, Layrargues G, Butterworth R. Increased densities of peripheral-type benzodiazepine receptors in brain autopsy samples from cirrhotic patients with hepatic encephalopathy. Hepatology. 1990;11(5):874-878. 
29. Cagnin A, Taylor-Robinson SD, Forton DM, Banati RB. In vivo imaging of cerebral "peripheral benzodiazepine binding sites" in patients with hepatic encephalopathy. Gut. 2006;55(4):547-553.

30. Shawcross D, Wright G, Olde Damink S, Jalan R. Role of ammonia and inflammation in minimal hepatic encephalopathy. Metab Brain Dis. 2007;22(1):125-138.

31. Liu Q, Duan Z, Ha D, Bengmark S, Kurtovic J, Riordan S. Synbiotic modulation of gut flora: effect on minimal hepatic encephalopathy in patients with cirrhosis. Hepatology. 2004;39(5):1441-1449.

32. Häussinger D, Kircheis G, Fischer R, Schliess F, Dahl S. Hepatic encephalopathy in chronic liver disease: a clinical manifestation of astrocyte swelling and low-grade cerebral edema? J Hepatol. 2000; 32(6):1035-1038.

33. Häussinger D, Laubenberger J, vom Dahl S, et al. Proton magnetic resonance spectroscopy studies on human brain Myo-inositol in hypoosmolarity and hepatic encephalopathy. Gastroenterology. 1994; 107(5):1475-1480.

34. Parsons-Smith B, Summerskill W, Dawson A, Sherlock S. The electroencephalograph in liver disease. Lancet. 1957;270(7001):867-871.

35. Conn HO, Leevy CM, Vlahcevic ZR, et al. Comparison of lactulose and neomycin in the treatment of chronic portal-systemic encephalopathy. A double blind controlled trial. Gastroenterology. 1977;72(4 pt 1): 573-583.

36. Zeegen R, Drinkwater J, Dawson A. Method for measuring cerebral dysfunction in patients with liver disease. BMJ. 1970;2(5710) 633-636.

37. Rikkers L, Jenko P, Rudman D, Freides D. Subclinical hepatic encephalopathy: detection, prevalence, and relationship to nitrogen metabolism. Gastroenterology. 1978;75(3):462-469.

38. Tarter RE, Hegedus AM, Van Thiel DH, Schade RR, Gavaler JS, Starzl TE. Nonalcoholic cirrhosis associated with neuropsychological dysfunction in the absence of overt evidence of hepatic encephalopathy Gastroenterology. 1984;86(6):1421-1427.

39. Ferenci P. Hepatic encephalopathy - definition, nomenclature, diagnosis, and quantification: final report of the working party at the 11th World Congresses of Gastroenterology, Vienna, 1998. Hepatology. 2002;35(3):716-721.

40. Groeneweg M, Quero JC, De Bruijn I, et al. Subclinical hepatic encephalopathy impairs daily functioning. Hepatology. 1998;28(1) 45-49.

41. Bergner M, Bobbitt R, Carter W, Gilson B. The sickness impact profile: development and final revision of a health status measure. Med Care. 1981;19(8):787-805.

42. Schomerus H, Hamster W. Quality of life in cirrhotics with minimal hepatic encephalopathy. Metab Brain Dis. 2001;16(1-2):37-41.

43. Wein C, Koch H, Popp B, Oehler G, Schauder P. Minimal hepatic encephalopathy impairs fitness to drive. Hepatology. 2004;39(3):739-745.

44. Bajaj J, Hafeezullah M, Hoffmann R, Saeian K. Minimal hepatic encephalopathy: a vehicle for accidents and traffic violations. $A m \mathrm{~J}$ Gastroenterol. 2007;102(9):1903-1909.

45. Groeneweg M, Moerland W, Quero J, Hop W, Krabbe P, Schalm S. Screening of subclinical hepatic encephalopathy. J Hepatol. 2000; 32(5):748-753.

46. Sood GK, Sarin SK, Mahaptra J, Broor SL. Comparative efficacy of psychometric tests in detection of subclinical hepatic encephalopathy in nonalcoholic cirrhotics: search for a rational approach. $\mathrm{Am} J$ Gastroenterol. 1989;84(2):156-159.

47. Marchesini G, Zoli M, Dondi C, et al. Prevalence of subclinical hepatic encephalopathy in cirrhotics and relationship to plasma amino acid imbalance. Digest Dis Sci. 1980;25(10):763-768.

48. Weissenborn K, Ennen J, Schomerus H, Rückert N, Hecker H. Neuropsychological characterization of hepatic encephalopathy. J Hepatol. 2001;34(5):768-773.

49. Reitan R. The relation of the trail making test to organic brain damage. J Consult Psychol. 1955;19(5):393-394.

50. Wechsler D. Wechsler Adult Intelligence Scale - Revised. New York: Psychological Corporation; 1981.
51. Romero Gómez M, Córdoba J, Jover R, et al. Normality tables in the spanish population for psychometric tests used in the diagnosis of minimal hepatic encephalopathy. Med Clin (Barc). 2006;127(7): 246-249.

52. Amodio P, Campagna F, Olianas S, et al. Detection of minimal hepatic encephalopathy: normalization and optimization of the psychometric hepatic encephalopathy score. A neuropsychological and quantified EEG study. J Hepatol. 2008;49(3):346-353.

53. Learmonth Y, Dlugonski D, Pilutti L, Sandroff B, Klaren R, Motl R. Psychometric properties of the fatigue severity scale and the modified fatigue impact scale. J Neurol Sci. 2013;331(1-2):102-107.

54. Prcic A, Aganovic D, Hadziosmanovic O. Sickness impact profile (SIP) score, a good alternative instrument for measuring quality of life in patients with ileal urinary diversions. Acta Informatica Medica. 2013; 21(3): 160

55. Treanor C, Donnelly M. A methodological review of the short form health survey 36 (SF-36) and its derivatives among breast cancer survivors. Qual Life Res. 2015;24(2):339-362.

56. Schatz P, Browndyke J. Applications of computer-based neuropsychological assessment. J Head Trauma Rehabil. 2002;17(5):395-410.

57. Tartaglione EV, Derleth M, Yu L, Ioannou GN. Can computerized brain training games be used to identify early cognitive impairment in cirrhosis? Am J Gastroenterol. 2014;109(3):316-323.

58. Foley JM, Watson CW, Adams RD. Significance of the electroencephalographic changes in hepatic coma. Trans Am Neurol Assoc. 1950;51: 161-165.

59. Kircheis G. Critical flicker frequency for quantification of low-grade hepatic encephalopathy. Hepatology. 2002;35(2):357-366.

60. Grover VP, Dresner MA, Forton DM, et al. Current and future applications of magnetic resonance imaging and spectroscopy of the brain in hepatic encephalopathy. World J Gastroenterol. 2006;12(19): 2969-2978.

61. Timmermann L, Butz M, Gross J, et al. Impaired cerebral oscillatory processing in hepatic encephalopathy. Neurophysiol Clin. 2008;119(2):265-272.

62. Dawson A. Neomycin in the treatment of hepatic coma. Lancet. 1957;270(7008):1263-1268.

63. Ferenci P, Herneth A, Steindl P. Newer approaches to therapy of hepatic encephalopathy. Semin Liver Dis. 1996;16(03):329-338.

64. Adachi J, DuPont H. Rifaximin: a novel nonabsorbed rifamycin for gastrointestinal disorders. Clin Infect Dis. 2006;42(4):541-547.

65. de Melo R, Charneski L, Hilas O. Rifaximin for the treatment of hepatic encephalopathy. Am J Health-Syst Pharm. 2008;65(9):818-822.

66. Bass NM, Mullen KD, Sanyal A, et al. Rifaximin treatment in hepatic encephalopathy. N Engl J Med. 2010;362(12):1071-1081.

67. Kimer N, Krag A, Moller S, Bendtsen F, Gluud LL. Systematic review with meta-analysis: the effects of rifaximin in hepatic encephalopathy. Aliment Pharmacol Ther. 2014;40(2):123-132.

68. Bircher J, Müller J, Guggenheim P, Haemmerli U. Treatment of chronic portal-systemic encephalopathy with lactulose. Lancet. 1966; 287(7443):890-893.

69. Weber FL Jr, Banwell JG, Fresard KM, Cummings JH. Nitrogen in fecal bacterial, fiber, and soluble fractions of patients with cirrhosis: effects of lactulose and lactulose plus neomycin. J Lab Clin Med. 1987; 110(3):259-263.

70. Butterworth R, Rose C, Quack G, Kircheis G. L-Ornithine-L-aspartate in experimental portal-systemic encephalopathy: therapeutic efficacy and mechanism of action. J Hepatol. 1998;28:70.

71. Rees C. Effect of L-ornithine-L-aspartate on patients with and without TIPS undergoing glutamine challenge: a double blind, placebo controlled trial. Gut. 2000;47(4):571-574.

72. Poo JL, Góngora J, Sánchez-Avila F, et al. Efficacy and safety of L-ornithine-L-aspartate in cirrhotic patients with hyperammonemic hepatic encephalopathy. Ann Hepatol. 2006;5(4):281-288.

73. Jiang Q, Jiang $\mathrm{X}$, Zheng $\mathrm{M}$, Chen Y. L-Ornithine-L-aspartate in the management of hepatic encephalopathy: a meta-analysis. $J$ Gastroenterol Hepatol. 2009;24(1):9-14. 
74. Prakash R, Mullen K. Mechanisms, diagnosis and management of hepatic encephalopathy. Nat Rev Gastroenterol Hepatol. 2010;7(9):515-525.

75. Riordan SM, Williams R. Treatment of hepatic encephalopathy. NEngl J Med. 1997;337(7):473-479.

76. Shukla S, Shukla A, Mehboob S, Guha S. Meta-analysis: the effects of gut flora modulation using prebiotics, probiotics and synbiotics on minimal hepatic encephalopathy. Aliment Pharmacol Ther. 2011;33(6): $662-671$.

77. Muto Y, Sato S, Watanabe A, et al. Effects of oral branched-chain amino acid granules on event-free survival in patients with liver cirrhosis. Clin Gastroenterol Hepatol. 2005;3(7):705-713.
78. Als-Nielsen B, Koretz RL, Kjaergard LL, Gluud C. Branched-chain amino acids for hepatic encephalopathy. Cochrane Database Syst Rev. 2003;(2):CD001939.

79. Neuberger J, Gimson A, Davies M, et al. Selection of patients for liver transplantation and allocation of donated livers in the UK. Gut. 2007; 57(2):252-257.

\section{Publish your work in this journal}

The International Journal of General Medicine is an international, peer-reviewed open-access journal that focuses on general and internal medicine, pathogenesis, epidemiology, diagnosis, monitoring and treatment protocols. The journal is characterized by the rapid reporting of reviews, original research and clinical studies across all disease areas.
A key focus is the elucidation of disease processes and management protocols resulting in improved outcomes for the patient. The manuscript management system is completely online and includes a very quick and fair peer-review system. Visit http://www.dovepress.com/ testimonials.php to read real quotes from published authors.

Submit your manuscript here: http://www.dovepress.com/international-journal-of-general-medicine-journal 